\title{
The reconstruction of a wave equation from one side measurement
}

\author{
Amin Boumenir and Vu Kim Tuan
}

\begin{abstract}
We are concerned with the reconstruction of a one dimensional wave equation, where the potential is known in a neighborhood of one of the end points of the boundary. We show then the sought potential can be determined by one single measurement of the solution at that end. We also show that the solution can be evaluated at the other end without the need of solving the wave equation.
\end{abstract}

\section{Introduction}

Consider the problem of recovering an unknown coefficient $q$ and constants $\ell, H$, appearing in the following wave equation

$$
\left\{\begin{array}{l}
u_{t t}(x, t)=u_{x x}(x, t)-q(x) u(x, t), \quad 0<x<\ell, \quad t>0, \\
u_{x}(0, t)-h u(0, t)=0, \quad u_{x}(\ell, t)+H u(\ell, t)=0, \quad t>0, \\
u(x, 0)=f(x) \quad \text { and } \quad u_{t}(x, 0)=g(x), \quad 0<x<\ell
\end{array}\right.
$$

This note is concerned with choosing initial conditions $\{f, g\}$ such that the data gathered from a single measurement of $u(0, t)$ for $t>0$ is enough for the reconstruction of the real valued function $q \in L(0, \ell)$. This will answer the question of how to recover $q$ when one of the endpoints on the boundary is not accessible for measurements, for example when dealing with graphs [1, 3], or clogged buried pipelines or blood vessels. To this end we require that

$$
h \in \mathbb{R} \text { is given, and } q(x) \text { is known for } 0<x<\epsilon \text {, where } \epsilon \text { is arbitrarily small. }
$$

In $[3$, it was shown that at most two measurements on both sides were required, i.e. two pairs of traces $\{u(0, t), u(\ell, t)\}$ that were generated by two special initial conditions. The issue here is to choose initial conditions $\{f, g\}$ in (1.1) such that the observation $u(0, t)$ carries all the necessary spectral data in order to apply the Gelfand-Levitan theory.

Statement of the problem: Assuming that (1.2) holds, can we reconstruct $q$, $\ell$, and $H$ from one single measurement of $\{u(0, t)\}_{t>0}$ ? Also can we compute $u(\ell, t)$ using $u(0, t)$ and $\{f, g\}$ only?

The main motivation behind the above setting is the problem of unclogging an inaccessible pipe such as a buried pipeline or a blood vessel, that can be accessed from one end only. The idea here is to locate the obstruction, i.e. $\ell$, and its type $H$, and then generate a pressure wave $u(\ell, t)$ that can dissolve it. This will avoid constructing $q$ and then solving the wave equation (1.1).

\section{Preliminaries}

The solution of (1.1) can be written as

$$
u(x, t)=\sum_{n \geq 0}\left(a\left(\lambda_{n}\right) \cos \left(t \sqrt{\lambda_{n}}\right)+b\left(\lambda_{n}\right) \frac{\sin \left(t \sqrt{\lambda_{n}}\right)}{\sqrt{\lambda_{n}}}\right) \frac{1}{\alpha_{n}^{2}} y\left(x, \lambda_{n}\right),
$$

2000 Mathematics Subject Classification. Primary 35R30, 35L05; Secondary 34A55 .

Key words and phrases. Wave equation, boundary inversion, Gelfand-Levitan theory. 
where the eigenfunctions $y\left(x, \lambda_{n}\right)$, for $n=0,1,2, \cdots$, are solutions of

$$
\left\{\begin{array}{l}
-y^{\prime \prime}(x, \lambda)+q(x) y(x, \lambda)=\lambda y(x, \lambda), \quad 0<x<\ell, \\
y(0, \lambda)=1, \quad y^{\prime}(0, \lambda)=h
\end{array}\right.
$$

and the eigenvalues, $\lambda_{0}, \lambda_{1}, \cdots$, are determined as the roots of $y^{\prime}(\ell, \lambda)+H y(\ell, \lambda)=0$. Denote their norms by $\alpha_{n}=\left\|y\left(x, \lambda_{n}\right)\right\|_{L^{2}(0, \ell)}$ and their Fourier coefficients by

$$
a\left(\lambda_{n}\right)=\int_{0}^{\ell} f(x) y\left(x, \lambda_{n}\right) d x, \quad b\left(\lambda_{n}\right)=\int_{0}^{\ell} g(x) y\left(x, \lambda_{n}\right) d x .
$$

If we assume that we are given $h$ and $q(x)$ for $0<x<\epsilon$, then we would know $y(x, \lambda)$ over the interval $[0, \epsilon]$. The transmutation operators, $[\mathbf{6}$, would also be known over the same interval

$$
\cos (x \sqrt{\lambda})=y(x, \lambda)+\int_{0}^{x} H(x, t) y(x, \lambda) d t, \quad 0<x<\epsilon .
$$

In order to extract the complete spectral data $\left\{\lambda_{n}, \alpha_{n}^{2}\right\}$ from (2.1) we need to ensure all the Fourier coefficients to satisfy $a^{2}\left(\lambda_{n}\right)+b^{2}\left(\lambda_{n}\right) \neq 0$. For simplicity we show the following proposition

Proposition 1. Given $q$ over $(0, \epsilon)$, where $0<\epsilon<1$ and $h \in \mathbb{R}$, we can construct a continuous function $g_{\epsilon}$ explicitly, such that

$$
\operatorname{supp}\left(g_{\epsilon}\right) \subset[0, \epsilon] \text { and } \int_{0}^{\ell} g_{\epsilon}(x) y(x, \lambda) d x \neq 0 \text { for all } \lambda \in \mathbb{R} \text {, }
$$

and in particular we have $b\left(\lambda_{n}\right) \neq 0$ for all $n \geq 0$.

Proof. Let $\psi(x)=(x-\epsilon)^{2} 1_{[0, \epsilon]}(x)$, where $1_{[0, \epsilon]}(x)=1$ if $x \in[0, \epsilon]$ and 0 otherwise. Its cosine transform, given by

$$
\int_{0}^{\ell} \psi(x) \cos (x \sqrt{\lambda}) d x=\int_{0}^{\epsilon}(\epsilon-x)^{2} \cos (x \sqrt{\lambda}) d x=2 \frac{\epsilon \sqrt{\lambda}-\sin (\epsilon \sqrt{\lambda})}{\lambda \sqrt{\lambda}}=O\left(\frac{1}{\lambda}\right), \quad \lambda \rightarrow \infty
$$

does not vanish for any $\lambda \in \mathbb{R}$, which is easy to see, since for $\lambda>0$ we have $\epsilon \sqrt{\lambda}>\sin (\epsilon \sqrt{\lambda})$, and if $\lambda \leq 0$, then the integrand is positive. Since the kernel $H(x, t)$, in (2.2), is known for $0<t \leq x<\epsilon$, we also have

$$
\begin{aligned}
0 \neq \int_{0}^{\ell} \psi(x) \cos (x \sqrt{\lambda}) d x & =\int_{0}^{\epsilon} \psi(x) y(x, \lambda) d x+\int_{0}^{\epsilon} \int_{0}^{x} H(x, t) y(t, \lambda) d t \psi(x) d x \\
& =\int_{0}^{\epsilon}\left\{\psi(t)+\int_{t}^{\epsilon} H(x, t) \psi(x) d x\right\} y(t, \lambda) d t
\end{aligned}
$$

Thus we can define the initial condition

$$
g_{\epsilon}(x)= \begin{cases}\psi(x)+\int_{x}^{\epsilon} H(t, x) \psi(t) d t, & 0<x<\epsilon \\ 0, & \epsilon \leq x<\ell\end{cases}
$$

which vanishes for $x>\epsilon$ and its $y$-transform, by (2.3), has no zeros for $\lambda \in \mathbb{R}$. This initial condition will essentially ensure that the Fourier coefficients $b\left(\lambda_{n}\right)$ do not vanish and are known explicitly

$$
b\left(\lambda_{n}\right)=\int_{0}^{\ell} g_{\epsilon}(x) y\left(x, \lambda_{n}\right) d x=2 \frac{\epsilon \sqrt{\lambda_{n}}-\sin \left(\epsilon \sqrt{\lambda_{n}}\right)}{\lambda_{n} \sqrt{\lambda_{n}}} \neq 0, \text { and } b\left(\lambda_{n}\right)=O\left(\frac{1}{\lambda_{n}}\right) .
$$

As for the initial condition $f$ in (1.1), we take $f(x)=0$, and so $a\left(\lambda_{n}\right)=0$. 


\section{Reading the data at $x=0$}

We now use the asymptotics of the eigenvalues $\lambda_{n} \sim\left(\frac{\pi}{\ell} n\right)^{2},\left[\mathbf{7}\right.$, of the norming constants $\alpha_{n}^{2} \sim \frac{\ell}{2}$, [7, and of the Fourier coefficients $b\left(\lambda_{n}\right)=O\left(\frac{1}{\lambda_{n}}\right)=O\left(\frac{1}{n^{2}}\right)$ of the initial condition defined by (2.4), to deduce that the observation

$$
u(0, t)=2 \sum_{n \geq 0} \frac{\epsilon \sqrt{\lambda_{n}}-\sin \left(\epsilon \sqrt{\lambda_{n}}\right)}{\lambda_{n}^{2}} \frac{\sin \left(t \sqrt{\lambda_{n}}\right)}{\alpha_{n}^{2}}
$$

is a continuous function in $t$, and by the Lebesgue dominated convergence theorem one can apply the Laplace transform to (3.1) termwise to obtain

$$
\mathcal{L}(u)(0, s)=\sum_{n \geq 0} \frac{\epsilon \sqrt{\lambda_{n}}-\sin \left(\epsilon \sqrt{\lambda_{n}}\right)}{\lambda_{n}^{2}} \frac{2 \sqrt{\lambda_{n}}}{\left(s^{2}+\lambda_{n}\right) \alpha_{n}^{2}}, \quad \Re(s)>0 .
$$

The series in (3.2) represents a meromorphic function, and one can use pole finding methods to read off all $\left\{ \pm i \sqrt{\lambda_{n}}\right\}$, i.e. all the eigenvalues that appear in (3.1) and then compute the residues of $\mathcal{L}(u)(0, s)$ at $\left\{i \sqrt{\lambda_{n}}\right\}$ to obtain the sequence

$$
\left\{\frac{\epsilon \sqrt{\lambda_{n}}-\sin \left(\epsilon \sqrt{\lambda_{n}}\right)}{i \lambda_{n}^{2} \alpha_{n}^{2}}\right\}_{n \geq 0}
$$

Recall that $\left\{\lambda_{n}\right\}$ are known from (3.2), and so we can deduce all the norming constants $\left\{\alpha_{n}^{2}\right\}_{n \geq 0}$ from (3.3). In other words we have the complete spectral data $\left\{\lambda_{n}, \alpha_{n}^{2}\right\}$ that is required to form the spectral function and so $q, \ell$ and $H$ by the Gelfand-Levitan theory, [6. It is known that asymptotics of the eigenvalues have the form $\mathbf{7}$ ]

$$
\sqrt{\lambda_{n}}=\frac{\pi}{\ell} n+\frac{1}{n \ell} a_{1}+o\left(\frac{1}{n}\right), \text { where } a_{1}=h+H+\frac{1}{2} \int_{0}^{\ell} q(x) d x .
$$

Thus taking the limits, we find

$$
\ell=\lim _{n \rightarrow \infty} \frac{n \pi}{\sqrt{\lambda_{n}}} \quad \text { and } \quad \lim _{n \rightarrow \infty} n\left(\ell \sqrt{\lambda_{n}}-n \pi\right)=h+H+\frac{1}{2} \int_{0}^{\ell} q(x) d x,
$$

and as we now know $q, \ell$ and $h$, we can get $H$. Thus we have proved

TheOREM 1. Assume that we know $h \in \mathbb{R}$ and $q(x)$ for $0<x<\epsilon$, with $\epsilon$ arbitrarily small. Then using the initial conditions $f=0, g=g_{\epsilon}$ as in (2.4), we can uniquely reconstruct $q$ over $(0, \ell)$ and $H$, from one single reading of $u(0, t)$ for $t>0$.

We next examine the limiting case $h \rightarrow \infty$, which is the Dirichlet boundary condition at $x=0$.

\section{Dirichlet condition}

We now briefly show that the above approach extends to the Dirichlet case

$$
\left\{\begin{array}{l}
u_{t t}(x, t)=u_{x x}(x, t)-q(x) u(x, t), \quad 0<x<\ell, \quad t>0, \\
u(0, t)=0, \quad u_{x}(\ell, t)+H u(\ell, t)=0, \quad t>0, \\
u(x, 0)=f(x) \quad \text { and } \quad u_{t}(x, 0)=g(x), \quad 0<x<\ell .
\end{array}\right.
$$

Since $u(0, t)=0$, we measure $u_{x}(0, t)$ for $t>0$, which is given by

$$
u_{x}(x, t)=\sum_{n \geq 0}\left(\widetilde{a}\left(\mu_{n}\right) \cos \left(t \sqrt{\mu_{n}}\right)+\widetilde{b}\left(\mu_{n}\right) \frac{\sin \left(t \sqrt{\mu_{n}}\right)}{\sqrt{\mu_{n}}}\right) \frac{1}{\widetilde{\alpha}_{n}^{2}} \varphi^{\prime}\left(x, \mu_{n}\right),
$$

where the eigenfunctions $\varphi\left(x, \mu_{n}\right)$, for $n=0,1,2, \cdots$, are solutions of

$$
\left\{\begin{array}{l}
-\varphi^{\prime \prime}(x, \mu)+q(x) \varphi(x, \mu)=\mu \varphi(x, \mu), \quad 0<x<\ell \\
\varphi(0, \mu)=0 \text { and } \varphi^{\prime}(0, \mu)=1 .
\end{array}\right.
$$


The eigenvalues $\mu_{n}$ are the roots of

$$
\varphi^{\prime}\left(\ell, \mu_{n}\right)+H \varphi\left(\ell, \mu_{n}\right)=0
$$

and the Fourier coefficients and norming constants are

$$
\widetilde{a}\left(\mu_{n}\right)=\int_{0}^{\ell} f(x) \varphi\left(x, \mu_{n}\right) d x, \quad \widetilde{b}\left(\mu_{n}\right)=\int_{0}^{\ell} g(x) \varphi\left(x, \mu_{n}\right) d x, \quad \widetilde{\alpha}_{n}=\left\|\varphi\left(x, \mu_{n}\right)\right\|_{L^{2}(0, \ell)} .
$$

If we again assume that we are given $q(x)$ for $0<x<\epsilon$, then we would know $\varphi(x, \mu)$ over the interval $[0, \epsilon]$ and so the transmutation operators, [5], is known explicitly

$$
\frac{\sin (x \sqrt{\mu})}{\sqrt{\mu}}=\varphi(x, \mu)+\int_{0}^{x} L(x, t) \varphi(t, \mu) d t, \quad 0<x<\epsilon .
$$

We now need the following proposition

Proposition 2. Given $q$ over $(0, \epsilon)$, where $0<\epsilon<1$, then we can construct a continuous function $\widetilde{g}_{\epsilon}$ explicitly, such that

$$
\operatorname{supp}\left(\widetilde{g}_{\epsilon}\right) \subset[0, \epsilon] \text { and } \int_{0}^{\ell} \widetilde{g}_{\epsilon}(x) \varphi(x, \mu) d x \neq 0 \text { for all } \mu \in \mathbb{R} .
$$

Proof. If we use again $\psi(x)=(\epsilon-x) 1_{[0, \epsilon]}(x)$, then its sine transform, given by

$$
\int_{0}^{\ell} \psi(x) \frac{1}{\sqrt{\mu}} \sin (x \sqrt{\mu}) d x=\int_{0}^{\epsilon}(\epsilon-x) \frac{1}{\sqrt{\mu}} \sin (x \sqrt{\mu}) d x=\frac{\epsilon \sqrt{\mu}-\sin (\epsilon \sqrt{\mu})}{\mu}=O\left(\frac{1}{\sqrt{\mu}}\right), \quad \mu \rightarrow \infty,
$$

does not vanish for any $\mu \in \mathbb{R}$. Since the kernel $L(x, t)$ in (4.3) is known for $0<t \leq x<\epsilon$, we also have

$$
\begin{aligned}
0 \neq \int_{0}^{\ell} \psi(x) \frac{1}{\sqrt{\mu}} \sin (x \sqrt{\mu}) d x & =\int_{0}^{\epsilon} \psi(x) \varphi(x, \mu) d x+\int_{0}^{\epsilon} \int_{0}^{x} L(x, t) \varphi(t, \mu) d t \psi(x) d x \\
& =\int_{0}^{\epsilon}\left\{\psi(t)+\int_{t}^{\epsilon} L(x, t) \psi(x) d x\right\} \varphi(t, \mu) d t .
\end{aligned}
$$

Thus, we define the initial condition

$$
\widetilde{g}_{\epsilon}(x)= \begin{cases}\psi(x)+\int_{x}^{\epsilon} L(t, x) \psi(t) d t, & 0<x<\epsilon \\ 0, & \epsilon \leq x<\ell\end{cases}
$$

which vanishes for $x>\epsilon$ and its $\varphi$-transform, by (4.4), has no zeros for $\mu \in \mathbb{R}$. This initial condition will essentially ensure that the Fourier coefficients $\widetilde{b}\left(\mu_{n}\right)$ do not vanish and are known explicitly

$$
\widetilde{b}\left(\mu_{n}\right)=\int_{0}^{\ell} \widetilde{g}_{\epsilon}(x) \varphi\left(x, \mu_{n}\right) d x=\frac{\epsilon \sqrt{\mu_{n}}-\sin \left(\epsilon \sqrt{\mu_{n}}\right)}{\mu_{n}} \neq 0 .
$$

Taking $f=0$, the observation $u_{x}(0, t)$ has the form

$$
u_{x}(0, t)=\sum_{n \geq 0} \frac{\epsilon \sqrt{\mu_{n}}-\sin \left(\epsilon \sqrt{\mu_{n}}\right)}{\mu_{n}^{3 / 2}} \frac{\sin \left(t \sqrt{\mu_{n}}\right)}{\widetilde{\alpha}_{n}^{2}},
$$

out of which we can extract the full spectral data $\left\{\mu_{n}, \widetilde{\alpha}_{n}\right\}_{n \geq 0}$. We have just proved

TheOREM 2. Assume that we know $q(x)$ for $0<x<\epsilon$, with $\epsilon$ arbitrarily small. Then using the initial conditions $f=0, g=\widetilde{g}_{\epsilon}$ as in (4.5), we can uniquely reconstruct $\ell, q$ over $(0, \ell)$ and $H$, from one single reading of $u_{x}(0, t)$ for $t>0$.

Proof. It remains to see that asymptotics for the eigenvalues $\mu_{n}$ are now given by 7 ]

$$
\sqrt{\mu_{n}}=\frac{\pi}{\ell} n+\frac{\pi}{2 \ell}+\frac{1}{n \ell} a_{1}+o\left(\frac{1}{n}\right), \text { where } a_{1}=H+\frac{1}{2} \int_{0}^{\ell} q(x) d x,
$$

which means

$$
\ell=\lim _{n \rightarrow \infty} \frac{n \pi}{\sqrt{\mu_{n}}} \quad \text { and } \quad H=\lim _{n \rightarrow \infty} n\left(\ell \sqrt{\mu_{n}}-\left(n+\frac{1}{2}\right) \pi\right)-\frac{1}{2} \int_{0}^{\ell} q(x) d x .
$$




\section{Determination of $u(\ell, t)$}

We consider the case of the wave equation (1.1), where $y(0, \lambda)=1$. Recall that recovered eigenvalues $\lambda_{n}$ are the zeros of the boundary function

$$
\Phi(\lambda)=y^{\prime}(\ell, \lambda)+H y(\ell, \lambda),
$$

which is an entire function of order $1 / 2$, and so can also be represented by its zeros by Hadamard factorization theorem [7]

$$
\Phi(\lambda)=\ell\left(\lambda_{0}-\lambda\right) \prod_{n \geq 1}\left(\frac{\ell^{2}}{\pi^{2} n^{2}}\left(\lambda_{n}-\lambda\right)\right) .
$$

So $\Phi(\lambda)$ is determined by the $\left\{\lambda_{n}\right\}_{n \geq 0}$ and to reconstruct $u(\ell, t)$ without the knowledge of $q$ we use the fact that

$$
y^{2}(x, \lambda)=\left(y^{\prime}(x, \lambda) \partial_{\lambda} y(x, \lambda)-y(x, \lambda) \partial_{\lambda} y^{\prime}(x, \lambda)\right)^{\prime},
$$

where $\partial_{\lambda}$ is the derivative with respect to $\lambda$, which yields

$$
\begin{aligned}
\alpha_{n}^{2} & =\int_{0}^{\ell}\left|y\left(x, \lambda_{n}\right)\right|^{2} d x \\
& =y^{\prime}\left(\ell, \lambda_{n}\right) \partial_{\lambda} y\left(\ell, \lambda_{n}\right)-y\left(\ell, \lambda_{n}\right) \partial_{\lambda} y^{\prime}\left(\ell, \lambda_{n}\right)-\left(y^{\prime}\left(0, \lambda_{n}\right) \partial_{\lambda} y\left(0, \lambda_{n}\right)-y\left(0, \lambda_{n}\right) \partial_{\lambda} y^{\prime}\left(0, \lambda_{n}\right)\right) \\
& =y^{\prime}\left(\ell, \lambda_{n}\right) \partial_{\lambda} y\left(\ell, \lambda_{n}\right)-y\left(\ell, \lambda_{n}\right) \partial_{\lambda} y^{\prime}\left(\ell, \lambda_{n}\right) \\
& =\Phi\left(\lambda_{n}\right) \partial_{\lambda} y\left(\ell, \lambda_{n}\right)-y\left(\ell, \lambda_{n}\right) \Phi^{\prime}\left(\lambda_{n}\right) \\
& =-y\left(\ell, \lambda_{n}\right) \Phi^{\prime}\left(\lambda_{n}\right)
\end{aligned}
$$

where we used the fact that $y(0, \lambda)=1, y^{\prime}(0, \lambda)=h$ and $\Phi\left(\lambda_{n}\right)=0$. Consequently,

$$
\frac{y\left(\ell, \lambda_{n}\right)}{\alpha_{n}^{2}}=-\Phi^{\prime}\left(\lambda_{n}\right)
$$

and therefore, using (2.1) and (2.5) we can deduce the profile $u(\ell, t)$

$$
u(\ell, t)=2 \sum_{n \geq 0} \frac{\sin \left(\epsilon \sqrt{\lambda_{n}}\right)-\epsilon \sqrt{\lambda_{n}}}{\lambda_{n}^{2}} \sin \left(t \sqrt{\lambda_{n}}\right) \Phi^{\prime}\left(\lambda_{n}\right)
$$

without the knowledge of $q$ or the integration of the wave equation (1.1).

\section{References}

[1] S. Avdonin and S. Nicaise, Source identification for the wave equation on graphs. C. R. Math. Acad. Sci. Paris 352 (2014), no. 11, 907-912.

[2] A. Boumenir and Vu Kim Tuan, Recovery of the heat coefficient by two measurements. Inverse Problems and Imaging 5 (2011), no. 4, 775-791.

[3] A. Boumenir and Vu Kim Tuan, An inverse problem for the wave equation. J. Inverse Ill-Posed Probl. 19 (2011), no. $4-5,573-592$.

[4] A. Kirsch, An introduction to the Mathematical Theory of Inverse Problems, Applied Mathematical Sciences 120, Springer, New York, 1996.

[5] B.M. Levitan, Inverse Sturm-Liouville Problems, VSP, 1987

[6] B. M. Levitan and M. G. Gasymov, Determination of a differential equation by two of its spectra. Russ. Math. Surveys 19 (1964), 1-62.

[7] V. A. Marčenko, Some questions in the theory of one-dimensional linear differential operators of the second order. American Mathematical Society Translations, Series 2. Vol. 101: Six Papers in Analysis, 1-104, American Mathematical Society, Providence, R.I., 1973.

Department of Mathematics,, University of West Georgia, Ga 30118, USA

E-mail address: boumenir@westga.edu, vu@westga.edu 